\title{
Determining conditions for best pollen quality of red-purple tree tomato (Solanum betaceum Cav.) germplasm
}

Andrea Sotomayor*, Jorge Merino, William Viera

DOI. 10.21931/RB/2021.06.04.14

Abstract: The germination and viability of pollen are characteristics required for fecundation when individuals of different or the same species are crossed. For this reason, assessing these parameters in selected individuals to be used in breeding programs will increase the chances for the obtainment of new progeny. In this study, pollen from different accessions of the red-purple tree tomato (Solanum betaceum Cav.) was used: local cultivar (Morado Puntón), two commercial varieties (Large Red and Oratia Red) and six segregants [(Solanum unilobum $\times$ Solanum betaceum) $\times$ Solanum betaceum]. Three types of flowers were taken (A-day of anthesis, B-one day after anthesis, and C-two days after anthesis). The pollen was conserved in two temperatures ( $4^{\circ}$ and $22^{\circ}$ C) and four storage times $(0,5,10,20$ days). The percentage of germination and pollen viability of the selected individuals were evaluated. It was observed that the commercial materials showed higher germination percentages than the segregants in flower $A$ and $B$ at a temperature of $4^{\circ} \mathrm{C}$ at all storage times, except for the segregants GT7P47 and GT7P48 at the same temperature on day 0 . In addition, high percentages of viability were obtained both in flowers $A$ and $B$, at both temperatures and at all storage times. However, the immediate use of pollen after it is collected is recommended because better germination is achieved. This study is helpful to improve breeding procedures in the initial stages of directed crosses.

Key words: Pollen quality, red-purple, tree tomato, Solanum betaceum, germplasm.

\section{Introduction}

Tree tomato (Solanum betaceum Cav.), native to the Andean region of South America ${ }^{1}$, belongs to the Solanaceae family, is popular in this region for its consumption in juices and as fresh fruit. This fruit is characterized by its slightly bitter, sour, and astringent taste with a particular aroma ${ }^{2,3}$. In Ecuador, the cultivated area is around 2,000 ha and produces 28,512 tons with a yield of $13.79 \mathrm{th} \mathrm{a}^{-1(4)}$. The cultivation of this fruit is carried out by small and medium producers ${ }^{5}$, but this fruit has excellent possibilities of positioning in the world market due to its excellent organoleptic characteristics, exotic aroma and flavor, and nutraceutical properties ${ }^{6}$.

In Ecuador, tree tomato production is based mainly on local cultivars, which are the product of natural crosses ${ }^{7}$. However, breeding processes have generated segregants, originating genotypes with significant phenotypic heterogeneity reflected in agronomic traits, fruit quality, and productivity ${ }^{5,8}$. Ecuadorian cultivars are not kept pure due to cross pollination that occurs in the production plots, generating fruits of different color, shape and size; being the most representative cultivars: Gigante Anaranjado, Anaranjado Puntón, Rendondo Anaranjado, Gigante Morado y Morado Puntón'; however, the cultivar Gigante Anaranjado is the one with the highest demand, consumption and production?

Breeding on this fruit crop is carried out because tree tomato presents yield limitations mainly due to the attack of pests ${ }^{10,11}$. On the other hand, this fruit has excellent nutritional and commercial value, causing desired relevant demand in the national and international market ${ }^{12}$. Furthermore, from the nutritional point of view, tree tomato fruit is an excellent source of vitamins $A, B 6, C$, and $E$, and minerals such as iron; it also has a low carbohydrate content and less than 40 calories per $100 \mathrm{~g}^{13}$; it is a valuable source of pectins that favor the preparation of jellies and jams ${ }^{14}$. In addition, it has compounds with antioxidant capacity such as lycopene, anthocyanins, and high content of polyphenols such as 3-0-caffeoylquinic acid and rosmarinic acid ${ }^{3,14}$
For those mentioned above, the Fruit Program of the National Agricultural Research Institute (INIAP) has evaluated tree tomato segregants with different levels of resistance and fruit quality (physical and chemical traits) ${ }^{5,8}$, to select elite materials to be used as parents in future crosses. Plant breeding through directed crosses is essential for generating new genotypes with better characteristics; consequently, pollen quality studies are essential in artificial hybridization techniques ${ }^{15,16}$ to guarantee fertilization and the generation of new progeny.

Determining the quality of pollen is of great importance in breeding ${ }^{17}$ because it has a tremendous impact in the efficacy of the genetic improvement practices ${ }^{18}$. This characteristic is essential to define the direction of a cross and have bases for the success of controlled hybridizations that guarantee the generation of new hybrids ${ }^{19}$, originating superior individuals with better productivity, fruit quality, and obtain resistance or tolerance pests.

Currently, the Fruit Program has identified tree tomato individuals with superior characteristics associated with fruit quality, including soluble solids content and red-purple mucilage, a characteristic related to a more significant amount of antioxidant compounds ${ }^{3}$. These individuals will be used as parents in future crosses in the breeding program; therefore, it is necessary to study the pollen quality to guarantee an appropriate starting material to continue with the breeding of this fruit crop successfully.

\section{Methods}

The research was carried out in the Laboratory of the Tumbaco Experimental Farm. The pollen belonged to 1 local cultivar (CMP4 -Morado Puntón), 2 commercial varieties (NZLRP5 - Large Red and NZORP7 - Oratia Red), and 6 segregants of tree tomato (GT7p47, GT7p48, GT9p18, GT20p2, GT20p7, and GT33p5) coming from the cross [Solanum unilobum $\mathrm{x}$ 
Solanum betceum] x Solanum betaceum ${ }^{5,8}$. These individuals were selected for their fruit quality characteristics (Table 1 , Figure 1).

The plants used for this study were grafted onto Nicotiana glauca, with an age of 4 years. They were sown in the Tumbaco Experimental Farm of the INIAP at an altitude of 2348 masl, with maximum temperatures of $27^{\circ} \mathrm{C}$ and minimum of $5^{\circ}$ C, precipitation of $800 \mathrm{~mm}$ per year, average relative humidity of $70.86 \%$, and geographical location of latitude: $00^{\circ} 13$ '00" South and longitude: $78^{\circ} 24^{\prime} 00^{\prime \prime}$ West.

The pollen was stored in two temperatures $\left(4^{\circ} \mathrm{C}\right.$ and $22^{\circ}$ C) and four periods of time $(0,5,10$, and 20 days $)$ for its conservation. For the 0-day storage period, storage was performed for 8 hours at the two temperatures. Pollen extraction was carried out in the morning (8:00 - 10:00 am), taking fully open flowers in 3 states (Figure 2): where flower A (day 0) is the day of anthesis, flower B (day 1 ) one day after anthesis and flower $\mathrm{C}$ (day 2) two days after anthesis. To extract the pollen, the tips of the anthers were cut, and the pollen of the flower was obtained using light strokes and shaking.

A $1 \mathrm{mg}$ sample of pollen was placed in Eppendorf tubes for each treatment. For the evaluation of pollen viability, the staining technique based on acetocarmine glycerol gelatin ${ }^{20}$ was used. This test measures the integrity of the cytoplasm; that is, the pollen grains turn red when the cytoplasmic membrane is intact ${ }^{21}$. Viable pollen was considered to be those grains that did not show deformations and had intense staining. While for the evaluation of pollen germination, the pollen was sown for 24 hours in a sucrose medium described by Rodríguez and Daf$\mathrm{ni}^{22}$. Germinated pollen grains were considered to be those that showed the pollen tube with a length greater than or equal to the pollen diameter ${ }^{23-25}$. In both cases, 250 pollen grains were counted using an optical microscope (Olympus, SX40).

A completely randomized design was used, with a $9 \times 2$ $\times 4 \times 3$ factorial arrangement with a total of 216 treatments with 5 observations. An analysis of variance was performed, and the 5\% Tukey test was used to determine differences between means.

\section{Results and discussion}

Research carried out on pollen quality is of great importance for the success of breeding programs aimed at the generation of hybrids with better agronomic characteristics that allow increasing the efficiency of genetic improvement through directed crosses $^{17}$.

\section{Germination}

To simulate pollen development in the gynoecium in vivo, the germination tests of pollen grains in vitro are established; this is achieved by placing the grains in a germination solution that must present similar conditions to the stigma of the female organ ${ }^{26}$. In terms of germination percentage (Figure 3), significant differences were observed in flower type and temperature as storage days progressed (Table 2, 3, and 4). In the tree tomato, the size and production of pollen grains varies ${ }^{7}$, and the type of flower to be collected is of utmost importance before pollen collection ${ }^{27}$. In this research, it was observed that flowers $A$ and $B$ showed values greater than $55 \%$ both at $22^{\circ} \mathrm{C}$ and $4^{\circ} \mathrm{C}$ on day $\mathrm{O}$, while in subsequent conservation times, values greater than $40 \%$ were observed only at $4^{\circ} \mathrm{C}$. On the other hand, in flower C, low values (less than $13 \%$ ) were obtained in the day 0 and the percentages were considerably reduced later until reach $0 \%$ in various individuals at 10 and 20 days. Consequently, while the storage time increases, the germination percentage decreases. This trend was also reported by Gonzáles et al. ${ }^{16}$ who found that in potato (S. tuberosum), pollen stored at a temperature of $17^{\circ} \mathrm{C}$ loses its germination percentage rapidly, but if the pollen is stored at $4^{\circ} \mathrm{C}$ it had up to $20 \%$ germination. Araméndiz et $a l^{17}$ also reported this behavior in a study carried out on eggplant (S. melongena), where the highest germination percentage was obtained at 0 days of storage and it decreased as the storage time progressed and the temperature increased.

Statistical differences between the tree tomato individuals were observed, highlighting the commercial individuals CMP4, NZLRP5, and NZORP7 with values in a range of 54 to

\begin{tabular}{|l|c|c|c|c|c|c|}
\hline Material & $\begin{array}{c}\text { Weight } \\
\text { of fruit } \\
\text { (g) }\end{array}$ & $\begin{array}{c}\text { Pulp } \\
\text { Color }\end{array}$ & $\begin{array}{c}\text { Color of } \\
\text { mucilage }\end{array}$ & $\begin{array}{c}\text { Content of } \\
\text { soluble } \\
\text { solids } \\
\text { Brix) }\end{array}$ & $\begin{array}{c}\text { Acidity } \\
\text { (\%) }\end{array}$ & $\begin{array}{c}\text { Firmness } \\
\text { (N) }\end{array}$ \\
\hline $\begin{array}{l}\text { CMP4 } \\
\text { Morado } \\
\text { Puntón }\end{array}$ & 92,56 & Orange & Red & 10,6 & 1,70 & 2,08 \\
\hline $\begin{array}{l}\text { NZLRP5 } \\
\text { Large } \\
\text { Red }\end{array}$ & 61,14 & Orange & Red & 11,74 & 1,31 & 2,77 \\
\hline $\begin{array}{l}\text { NZORP7 } \\
\text { Oratia } \\
\text { Red }\end{array}$ & 70,28 & Orange & Red & 9,92 & 1,26 & 1,34 \\
\hline GT7p47 & 50,49 & Orange & Purple & 11,03 & 2,47 & 2,19 \\
\hline GT7p48 & 59,31 & Orange & Red & 11,02 & 1,78 & 1,87 \\
\hline GT9p18 & 66,97 & Orange & Purple & 12,64 & 1,31 & 1,83 \\
\hline GT20p2 & 84,24 & Orange & Purple & 11,92 & 2,52 & 1,25 \\
\hline GT20p7 & 80,17 & Orange & Purple & 12,03 & 2,37 & 1,38 \\
\hline GT33p5 & 41,10 & Orange & Purple & 11,10 & 1,25 & 3,10 \\
\hline
\end{tabular}

Table 1. Characteristics of the tree tomato germplasm. 

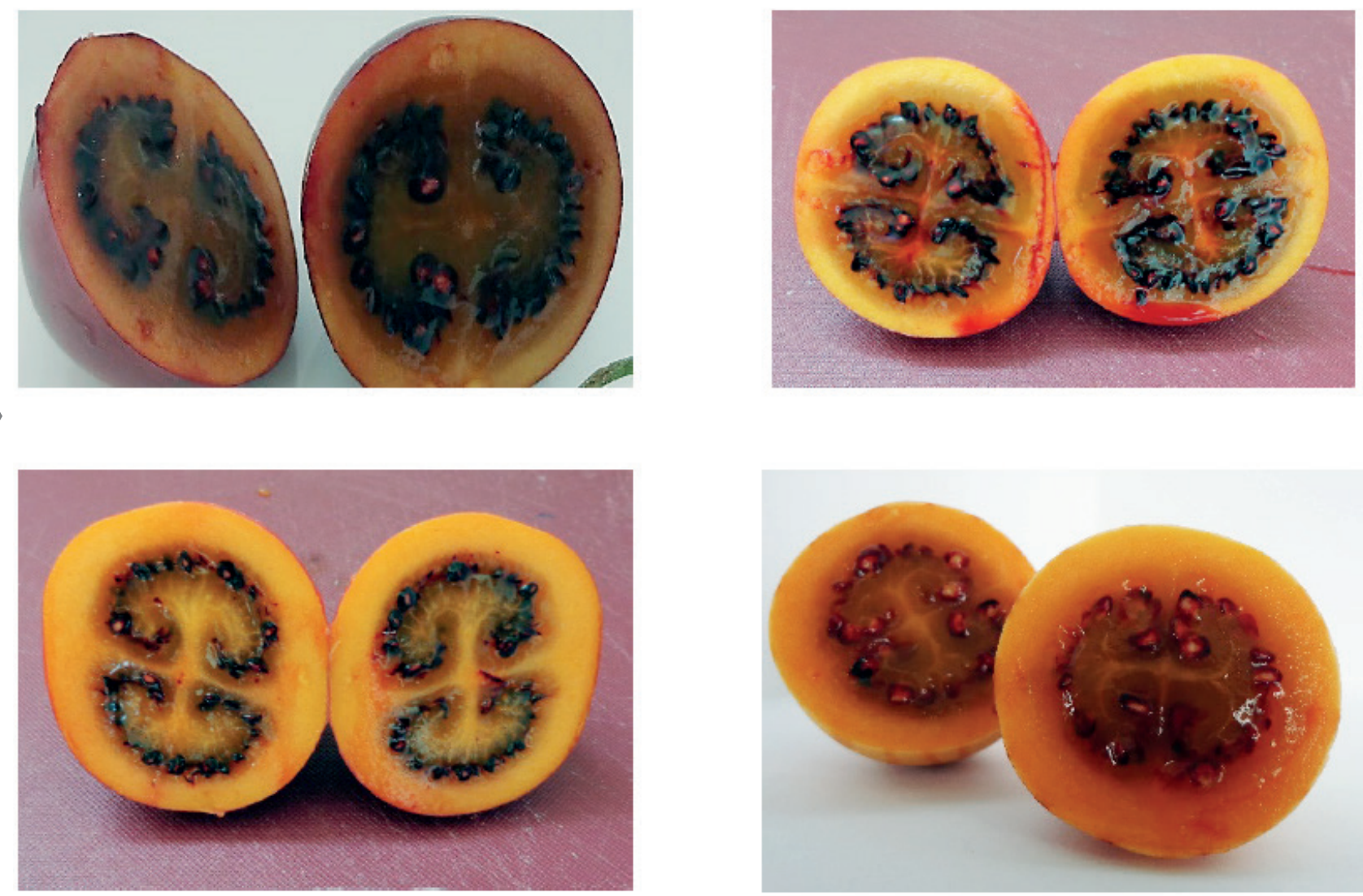

Figure 1. Tree tomato materials. Variety Large Red (top left), cultivar Morado Puntón (top right), and segregants (bottom left and right).

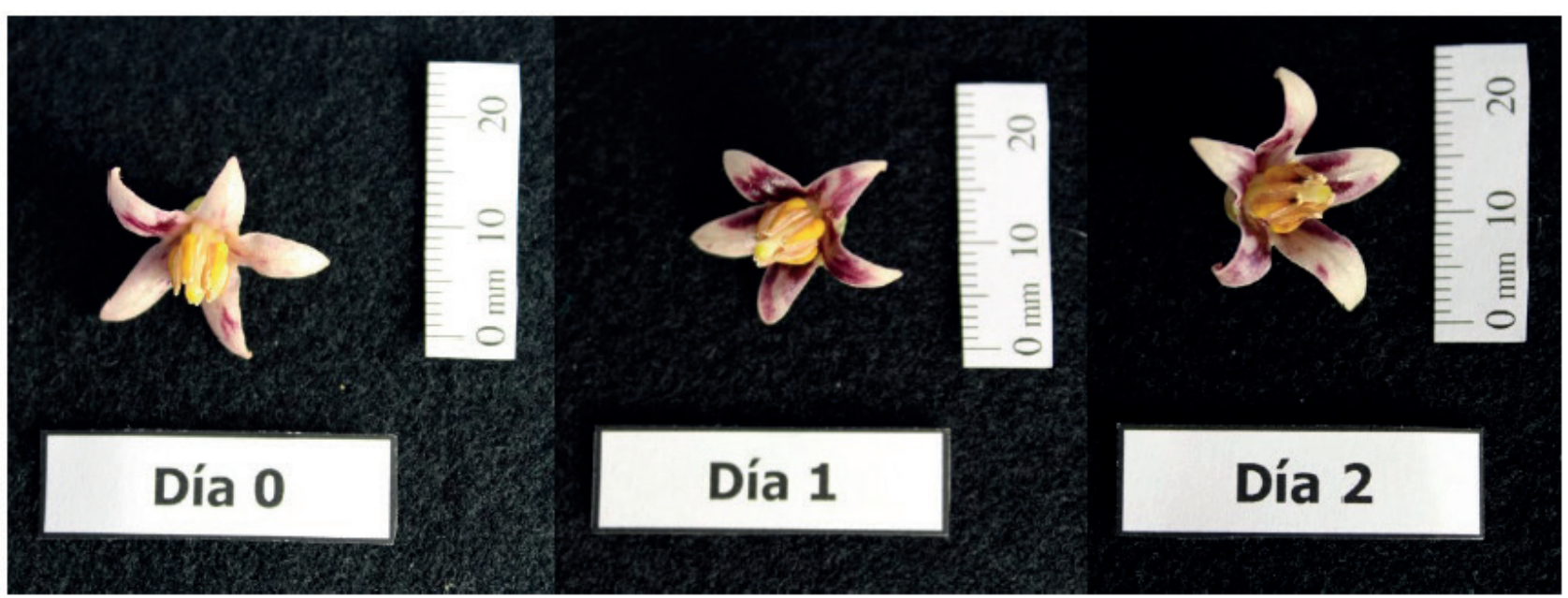

Figure 2. Flower types, where flower day 0 is the day of anthesis, flower day 1 is one day after anthesis, and flower day 2 is two days after anthesis.

$59 \%$ at $22^{\circ} \mathrm{C}$ and 53 to $64 \%$ at $4^{\circ} \mathrm{C}$ at $\mathrm{O}$ days of storage in flower $\mathrm{A}$. The germination percentage was not optimum (less than $70 \%$ ), even at $4^{\circ} \mathrm{C}$, which is a temperature commonly used to conserve pollen grains in some species. Later these values decreased, but CMP4 and NZLRP5 showed higher percentages, around 42\%, at 20 days (Table 2). Only the GT7P47 and GT7P48 segregants showed germination percentages higher than $53 \%$ at $22^{\circ} \mathrm{C}$ and $4^{\circ} \mathrm{C}$ at 0 days and around $42 \%$ at 5 days at $4^{\circ} \mathrm{C}$; while at 20 days, their germination decreased to values of 16 and $26 \%$ respectively (Table 2 ). For flower $B$, the trend was similar to that observed in flower $\mathrm{A}$ with germination percentages in a range between 50 and $59 \%$ at 0 days and decreasing considerably until 20 days (Table 3 ). In flower $\mathrm{C}$, only the segregant GT2OP7 had values of $12 \%\left(22^{\circ} \mathrm{C}\right)$ at 0 days and $9 \%\left(4^{\circ} \mathrm{C}\right)$ at 5 days, while the rest of the individuals showed shallow values or did not have germination (Table 4). According to Revelo et $a L^{28}$, this response is because commercial cultivars or varieties are better adapted to climatic and soil conditions, showing a more significant number of fruit set per inflorescence, corroborating that pollen from commercial materials has a superior germination capacity.

\section{Pollen viability}

The determination of pollen viability allows making reliable fertility estimates, besides being used for incompatibility studies in crosses $^{29}$. In fruit crops, staining with $2 \%$ acetocarmine and observation by optical microscope are used to de- 
termine the viability of the pollen grain ${ }^{26}$ (Figure 3). Similar to germination, significant differences were observed for the type of flower and temperature as the storage time advanced.

Flower $A$ and $B$ showed high percentages of viability (greater than 95\%) (Table 2 and 3) in the commercial materials CMP4, NZLRP5, and NZORP7, as well as in the segregants GT7P 47 and GT7P48, both at $22^{\circ} \mathrm{C}$ and at $4^{\circ} \mathrm{C}$ and at all storage times $(0,5,10$ and 20 days). While in flower $\mathrm{C}$, only the segregating GT20P7 obtained $12 \%$ viability at $22^{\circ} \mathrm{C}$ at $\mathrm{O}$ days and $8 \%$ at $4^{\circ} \mathrm{C}$ at 5 days, while the rest of the individuals showed shallow values, most of which did not have viability (Table 4). The results obtained in flower $C$ corroborate what Montaner et al. ${ }^{31}$ found that pollen loses its viability after its anthesis period.

In other Solanaceae species, using the same method of this research, viability percentages have been reported chiefly over $80 \%$ and reaching up to $98 \% 26,30$. On the other hand, in a study of pollen quality in chirimoya (Annona cherimola), it was reported that in storage at $7^{\circ} \mathrm{C}$, higher viability percentages are achieved as the conservation time advances ${ }^{32}$. This study obtained stable percentages throughout the pollen storage time at the two temperatures evaluated (Table 2 and 3).
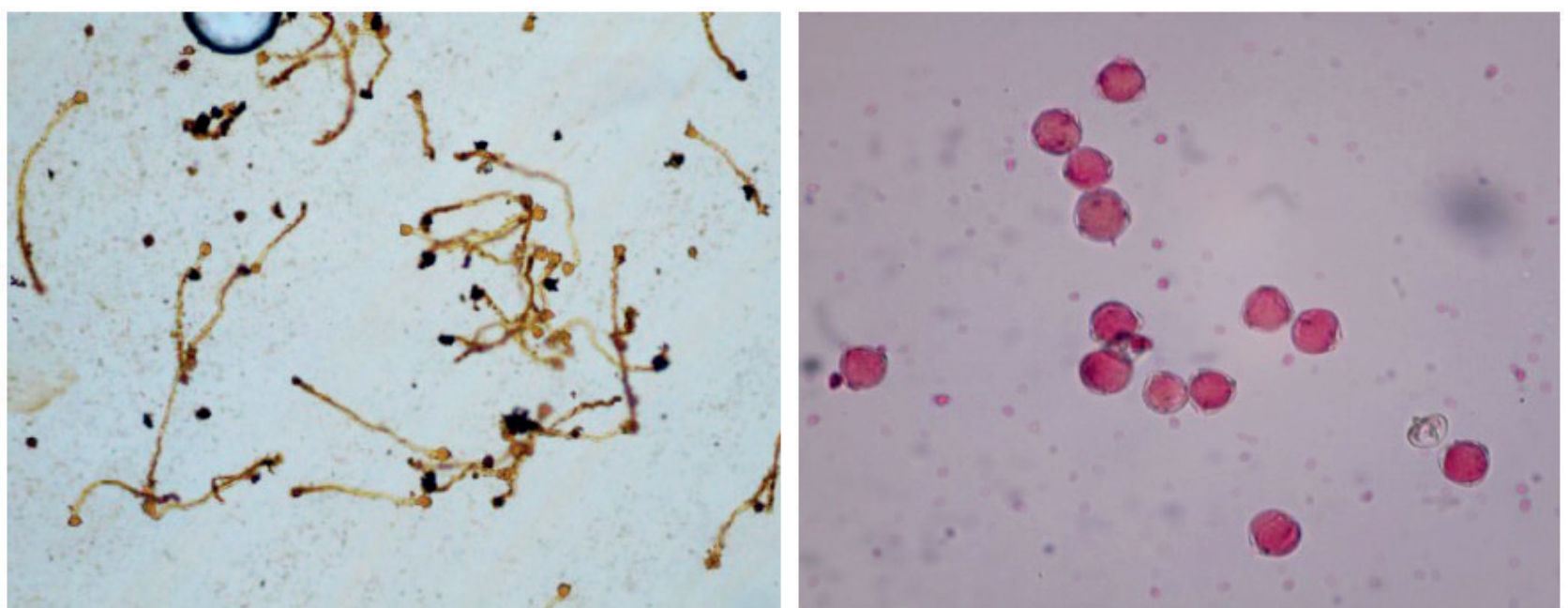

Figure 3. Flower $\mathrm{A}$, day 0 , temperature $22^{\circ}$. Germination test, arrow indicates germinated pollen tube (left). Pollen viability test by acetocarmin staining (right)

\begin{tabular}{|c|c|c|c|c|c|c|c|c|}
\hline \multirow{2}{*}{$\begin{array}{l}\text { Number of days } \\
\text { Temperature }\end{array}$} & \multicolumn{2}{|c|}{ od } & \multicolumn{2}{|c|}{$5 d$} & \multicolumn{2}{|c|}{$10 \mathrm{~d}$} & \multicolumn{2}{|c|}{ 20d } \\
\hline & $22^{\circ} \mathrm{C}$ & $4^{\circ} \mathrm{C}$ & $22^{\circ} \mathrm{C}$ & $4^{\circ} \mathrm{C}$ & $22^{\circ} \mathrm{C}$ & $4^{\circ} \mathrm{C}$ & $22^{\circ} \mathrm{C}$ & $4^{\circ} \mathrm{C}$ \\
\hline \multicolumn{9}{|l|}{ Germination (\%) } \\
\hline CMP4 & $59.76 \pm 0.69 \mathrm{a}$ & $64.61 \pm 1.24 \mathrm{a}$ & $8.98 \pm 0.89 \mathrm{~b}$ & $58.63 \pm 1.50 \mathrm{a}$ & $3.09 \pm 1.09 \mathrm{c}$ & $55.71 \pm 0.96 \mathrm{a}$ & $0.24 \pm 0.36 \mathrm{~b}$ & $41.53 \pm 2.46 \mathrm{a}$ \\
\hline GT20P2 & $1.71 \pm 2.14 \mathrm{~d}$ & $0.32 \pm 0.34 \mathrm{~d}$ & $0.95 \pm 1.10 \mathrm{c}$ & $1.35 \pm 0.83 \mathrm{~d}$ & $0.40 \pm 0.28 \mathrm{~d}$ & $0.64 \pm 0.36 \mathrm{f}$ & $0.00 \pm 0.00 \mathrm{~b}$ & $0.32 \pm 0.33 \mathrm{f}$ \\
\hline GT20P7 & $34.82 \pm 2.05 b$ & $18.31 \pm 2.16 \mathrm{c}$ & $16.18 \pm 2.18 \mathrm{a}$ & $22.68 \pm 2.71 c$ & $8.16 \pm 1.39 \mathrm{a}$ & $14.98 \pm 1.34 \mathrm{e}$ & $4.95 \pm 0.88 \mathrm{a}$ & $5.97 \pm 2.20 \mathrm{e}$ \\
\hline GT33P5 & $21.42 \pm 1.28 \mathrm{c}$ & $14.90 \pm 1.22 c$ & $14.29 \pm 1.77 \mathrm{a}$ & $20.62 \pm 1.47 c$ & $3.68 \pm 2.69 b c$ & $13.11 \pm 1.51 \mathrm{e}$ & $0.56 \pm 0.46 \mathrm{~b}$ & $0.80 \pm 0.80 \mathrm{f}$ \\
\hline GT7P47 & $56.4 \pm 3.14 \mathrm{a}$ & $57.46 \pm 1.22 \mathrm{ab}$ & $7.64 \pm 1.79 \mathrm{~b}$ & $41.57 \pm 1.68 \mathrm{~b}$ & $2.06 \pm 0.94 \mathrm{~cd}$ & $35.94 \pm 1.81 \mathrm{~d}$ & $0.24 \pm 0.22 \mathrm{~b}$ & $16.68 \pm 1.63 \mathrm{~d}$ \\
\hline GT7P48 & $55.52 \pm 3.78 \mathrm{a}$ & $53.43 \pm 3.31 \mathrm{~b}$ & $7.04 \pm 1.58 \mathrm{~b}$ & $43.6 \pm 3.23 \mathrm{~b}$ & $3.63 \pm 0.76 b c$ & $40.50 \pm 1.33 c$ & $0.56 \pm 0.22 \mathrm{~b}$ & $26.19 \pm 1.73 c$ \\
\hline GT9P18 & $3.63 \pm 3.54 \mathrm{~d}$ & $1.02 \pm 0.57 \mathrm{~d}$ & $0.16 \pm 0.21 \mathrm{c}$ & $1.25 \pm 0.91 \mathrm{~d}$ & $0.32 \pm 0.33 \mathrm{~d}$ & $0.56 \pm 0.36 \mathrm{f}$ & $0.00 \pm 0.00 \mathrm{~b}$ & $0.31 \pm 0.32 \mathrm{f}$ \\
\hline NZLRP5 & $55.44 \pm 2.02 \mathrm{a}$ & $62.37 \pm 8.39 \mathrm{a}$ & $8.12 \pm 1.94 b$ & $42.13 \pm 4.21 \mathrm{~b}$ & $2.69 \pm 1.41 \mathrm{~cd}$ & $52.96 \pm 2.11 \mathrm{a}$ & $0.24 \pm 0.22 \mathrm{~b}$ & $43.36 \pm 1.54 \mathrm{a}$ \\
\hline NZORP7 & $54.71 \pm 2.73 \mathrm{a}$ & $58.67 \pm 6.47 \mathrm{ab}$ & $9.72 \pm 1.00 \mathrm{~b}$ & $54.29+2.61 \mathrm{a}$ & $5.95 \pm 0.92 \mathrm{ab}$ & $44.70 \pm 1.84 \mathrm{~b}$ & $0.72 \pm 0.33 \mathrm{~b}$ & $36.16 \pm 0.48 \mathrm{~b}$ \\
\hline \multicolumn{9}{|l|}{ Viability (\%) } \\
\hline CMP4 & $97.68 \pm 1.43 \mathrm{a}$ & $97.12 \pm 1.04 \mathrm{a}$ & $97.04 \pm 1.40 \mathrm{a}$ & $98.80 \pm 0.49 \mathrm{a}$ & $97.04 \pm 1.51 \mathrm{a}$ & $97.92 \pm 1.51 \mathrm{a}$ & $97.12 \pm 1.07 \mathrm{a}$ & $97.92 \pm 1.51 \mathrm{ab}$ \\
\hline GT20P2 & $1.35 \pm 0.92 \mathrm{c}$ & $0.17 \pm 0.23 \mathrm{c}$ & $0.80 \pm 0.80 \mathrm{c}$ & $1.05 \pm 0.86 \mathrm{e}$ & $0.24 \pm 0.22 c$ & $0.48 \pm 0.44 c$ & $0.00 \pm 0.00 \mathrm{~b}$ & $0.16 \pm 0.22 \mathrm{~d}$ \\
\hline GT20P7 & $33.51 \pm 3.17 \mathrm{~b}$ & $17.63 \pm 1.99 \mathrm{~b}$ & $12.15 \pm 2.09 \mathrm{~b}$ & $24.46 \pm 3.16 c$ & $4.80 \pm 1.95 b$ & $13.76 \pm 2.63 b$ & $0.48 \pm 0.52 b$ & $6.21 \pm 2.37 c$ \\
\hline GT33P5 & $0.00 \pm 0.00 \mathrm{c}$ & $14.97 \pm 2.99 \mathrm{~b}$ & $0.00 \pm 0.00 \mathrm{c}$ & $19.39 \pm 1.41 \mathrm{~d}$ & $0.00 \pm 0.00 \mathrm{c}$ & $0.00 \pm 0.00 \mathrm{c}$ & $0.00 \pm 0.00 \mathrm{~b}$ & $0.00 \pm 0.00 \mathrm{~d}$ \\
\hline GT7P47 & $97.76 \pm 1.43 \mathrm{a}$ & $97.52 \pm 1.25 \mathrm{a}$ & $97.44 \pm 1.46 \mathrm{a}$ & $97.92 \pm 1.34 \mathrm{ab}$ & $98.08 \pm 1.04 \mathrm{a}$ & $97.60 \pm 1.36 \mathrm{a}$ & $98.08 \pm 1.43 \mathrm{a}$ & $97.92 \pm 1.51 \mathrm{ab}$ \\
\hline GT7P48 & $98.16 \pm 1.08 \mathrm{a}$ & $97.04 \pm 1.28 \mathrm{a}$ & $98.40 \pm 1.26 \mathrm{a}$ & $98.00 \pm 1.10 \mathrm{a}$ & $97.60 \pm 1.33 \mathrm{a}$ & $97.36 \pm 1.28 \mathrm{a}$ & $97.76 \pm 1.43 \mathrm{a}$ & $97.60 \pm 1.33 \mathrm{ab}$ \\
\hline GT9P18 & $2.03 \pm 2.45 c$ & $1.56 \pm 1.03 \mathrm{c}$ & $0.40 \pm 0.49 c$ & $0.70 \pm 0.64 \mathrm{e}$ & $0.64 \pm 0.54 \mathrm{c}$ & $0.16 \pm 0.21 \mathrm{c}$ & $0.24 \pm 0.22 \mathrm{~b}$ & $0.16 \pm 0.21 \mathrm{~d}$ \\
\hline NZLRP5 & $96.48 \pm 1.58 \mathrm{a}$ & $98.24 \pm 1.73 \mathrm{a}$ & $98.40 \pm 1.26 \mathrm{a}$ & $95.60 \pm 0.85 b$ & $97.20 \pm 1.36 \mathrm{a}$ & $98.24 \pm 1.28 \mathrm{a}$ & $98.40 \pm 1.26 \mathrm{a}$ & $98.48 \pm 1.34 \mathrm{a}$ \\
\hline NZORP7 & $97.76 \pm 1.31 \mathrm{a}$ & $98.48 \pm 15.93 \mathrm{a}$ & $97.44 \pm 1.61 \mathrm{a}$ & $99.36 \pm 0.61 \mathrm{a}$ & $98.00 \pm 1.41 \mathrm{a}$ & $95.60 \pm 0.85 \mathrm{a}$ & $97.92 \pm 1.73 \mathrm{a}$ & $95.76 \pm 0.96 \mathrm{ab}$ \\
\hline
\end{tabular}

Table 2. Pollen germination and viability in Flower $A$ as a function of tree tomato plant material, number of days and temperature. 


\begin{tabular}{|c|c|c|c|c|c|c|c|c|}
\hline \multirow{2}{*}{$\begin{array}{l}\text { Number of days } \\
\text { Temperature }\end{array}$} & \multicolumn{2}{|c|}{ od } & \multicolumn{2}{|c|}{$5 d$} & \multicolumn{2}{|c|}{$10 \mathrm{~d}$} & \multicolumn{2}{|c|}{ 20d } \\
\hline & $22^{\circ} \mathrm{C}$ & $4^{\circ} \mathrm{C}$ & $22^{\circ} \mathrm{C}$ & $4^{\circ} \mathrm{C}$ & $22^{\circ} \mathrm{C}$ & $4^{\circ} \mathrm{C}$ & $22^{\circ} \mathrm{C}$ & $4^{\circ} \mathrm{C}$ \\
\hline \multicolumn{9}{|l|}{ Germination (\%) } \\
\hline CMP4 & $55.52 \pm 3.78 \mathrm{a}$ & $58.04 \pm 1.52 \mathrm{ab}$ & $7.35 \pm 2.01 \mathrm{a}$ & $54.99 \pm 1.18 \mathrm{a}$ & $3.63 \pm 0.76 b$ & $50.76 \pm 1.04 \mathrm{a}$ & $0.56 \pm 0.22 \mathrm{ab}$ & $39.57 \pm 1.77 \mathrm{a}$ \\
\hline GT20P2 & $0.72 \pm 0.33 \mathrm{c}$ & $0.24 \pm 0.22 \mathrm{e}$ & $0.24 \pm 0.35 b$ & $0.56 \pm 0.36 \mathrm{e}$ & $0.16 \pm 0.22 \mathrm{c}$ & $0.24 \pm 0.36 \mathrm{f}$ & $0.00 \pm 0.00 \mathrm{c}$ & $0.48 \pm 0.33 \mathrm{e}$ \\
\hline GT20P7 & $21.83 \pm 1.93 \mathrm{~b}$ & $10.63 \pm 2.03 \mathrm{~d}$ & $9.40 \pm 1.35 \mathrm{a}$ & $12.4 \pm 1.89 \mathrm{~d}$ & $0.24 \pm 0.22 c$ & $5.34 \pm 1.05 \mathrm{e}$ & $0.00 \pm 0.00 \mathrm{c}$ & $0.24 \pm 0.22 \mathrm{e}$ \\
\hline GT33P5 & $4.21 \pm 3.41 \mathrm{c}$ & $1.04 \pm 1.19 \mathrm{e}$ & $0.00 \pm 0.00 \mathrm{~b}$ & $1.65 \pm 2.04 \mathrm{e}$ & $0.00 \pm 0.00 \mathrm{c}$ & $2.57 \pm 2.33$ ef & $0.00 \pm 0.00 \mathrm{c}$ & $0.72 \pm 0.33 \mathrm{e}$ \\
\hline GT7P47 & $54.71 \pm 2.73 \mathrm{a}$ & $55.23 \pm 1.07 \mathrm{ab}$ & $8.07 \pm 1.46 \mathrm{a}$ & $36.11 \pm 0.62 c$ & $2.06 \pm 0.94 b$ & $32.32 \pm 1.22 \mathrm{~d}$ & $0.24 \pm 0.22 \mathrm{bc}$ & $14.37 \pm 0.93 \mathrm{~d}$ \\
\hline GT7P48 & $55.44 \pm 2.02 \mathrm{a}$ & $50.21 \pm 3.35 \mathrm{c}$ & $9.72 \pm 1.00 \mathrm{a}$ & $38.86 \pm 1.20 \mathrm{c}$ & $2.69 \pm 1.41 \mathrm{~b}$ & $36.08 \pm 1.17 \mathrm{c}$ & $0.72 \pm 0.33 \mathrm{a}$ & $24.17 \pm 5.67 c$ \\
\hline GT9P18 & $0.00 \pm 0.00 \mathrm{c}$ & $0.24 \pm 0.22 \mathrm{e}$ & $0.00 \pm 0.00 \mathrm{~b}$ & $0.16 \pm 0.21 \mathrm{e}$ & $0.00 \pm 0.00 \mathrm{c}$ & $0.00 \pm 0.00 \mathrm{f}$ & $0.00 \pm 0.00 \mathrm{c}$ & $0.00 \pm 0.00 \mathrm{e}$ \\
\hline NZLRP5 & $55.89 \pm 3.48 \mathrm{a}$ & $59.06 \pm 1.25 \mathrm{a}$ & $7.64 \pm .1 .79 \mathrm{a}$ & $37.66 \pm 2.19 \mathrm{c}$ & $2.06 \pm 0.94 \mathrm{~b}$ & $48.59 \pm 2.72 \mathrm{a}$ & $0.24 \pm 0.22 \mathrm{bc}$ & $41.75 \pm 1.66 \mathrm{a}$ \\
\hline NZORP7 & $54.71 \pm 2.73 \mathrm{a}$ & $53.58 \pm 4.69 \mathrm{bc}$ & $9.72 \pm 1.00 \mathrm{a}$ & $49.22 \pm 1.07 \mathrm{~b}$ & $6.25 \pm 0.83 \mathrm{a}$ & $40.48 \pm 1.14 b$ & $0.72 \pm 0.33 \mathrm{a}$ & $34.43 \pm 0.64 b$ \\
\hline \multicolumn{9}{|l|}{ Viability (\%) } \\
\hline CMP4 & $98.16 \pm 1.08 \mathrm{a}$ & $97.12 \pm 1.04 \mathrm{a}$ & $98.40 \pm 1.26 \mathrm{a}$ & $98.80 \pm 0.49 \mathrm{a}$ & $98.00 \pm 1.41 \mathrm{a}$ & $97.92 \pm 1.51 \mathrm{a}$ & $97.12 \pm 1.07 \mathrm{a}$ & $97.92 \pm 1.51 \mathrm{a}$ \\
\hline GT20P2 & $0.56 \pm 0.45 c$ & $0.24 \pm 0.22 \mathrm{c}$ & $0.16 \pm 0.22 \mathrm{c}$ & $0.24 \pm 0.36 \mathrm{~d}$ & $0.08 \pm 0.18 \mathrm{~b}$ & $0.32 \pm 0.18 \mathrm{c}$ & $0.16 \pm 0.22 \mathrm{~b}$ & $0.08 \pm 0.18 \mathrm{c}$ \\
\hline GT20P7 & $21.08 \pm 1.29 \mathrm{~b}$ & $10.67 \pm 2.53 \mathrm{c}$ & $6.88 \pm 2.18 b$ & $6.53 \pm 1.38 c$ & $0.16 \pm 0.22 \mathrm{~b}$ & $0.48 \pm 0.44 \mathrm{c}$ & $0.00 \pm 0.00 \mathrm{~b}$ & $0.08 \pm 0.18 c$ \\
\hline GT33P5 & $0.88 \pm 0.71 c$ & $0.76 \pm 0.59 \mathrm{c}$ & $0.00 \pm 0.00 \mathrm{c}$ & $2.20 \pm 2.32 \mathrm{~d}$ & $0-00 \pm 0.00 \mathrm{~b}$ & $1.51 \pm 1.42 \mathrm{c}$ & $0.00 \pm 0.00 \mathrm{~b}$ & $0.40 \pm 0.49 c$ \\
\hline GT7P47 & $97.76 \pm 1.43 \mathrm{a}$ & $98.48 \pm 15.93 \mathrm{a}$ & $97.44 \pm 1.46 \mathrm{a}$ & $98.00 \pm 1.10 \mathrm{a}$ & $97.60 \pm 1.33 a$ & $95.60 \pm 0,85 b$ & $97.76 \pm 1.43 \mathrm{a}$ & $97.92 \pm 1.51 \mathrm{a}$ \\
\hline GT7P48 & $97.76 \pm 0.43 \mathrm{a}$ & $97.52 \pm 1.25 \mathrm{a}$ & $97.44 \pm 1.61 \mathrm{a}$ & $99.36 \pm 0.61 \mathrm{a}$ & $98.08 \pm 1.04 \mathrm{a}$ & $97.36 \pm 1.28 \mathrm{ab}$ & $97.92 \pm 1.73 \mathrm{a}$ & $98.48 \pm 1.34 \mathrm{a}$ \\
\hline GT9P18 & $0.48 \pm 0.43 c$ & $0.32 \pm 0.18 \mathrm{c}$ & $0.16 \pm 0.22 \mathrm{c}$ & $0.48 \pm 0.44 \mathrm{~d}$ & $0.08 \pm 0.18 \mathrm{~b}$ & $0.08 \pm 0.18 \mathrm{c}$ & $0.00 \pm 0.00 \mathrm{~b}$ & $0.00 \pm 0.00 \mathrm{c}$ \\
\hline NZLRP5 & $96.48 \pm 1.58 \mathrm{a}$ & $98.24 \pm 1.73 \mathrm{a}$ & $98.40 \pm 1.26 \mathrm{a}$ & $95.60 \pm 0.85 b$ & $97.20 \pm 1.36 \mathrm{a}$ & $98.24 \pm 1.28 \mathrm{a}$ & $98.40 \pm 1.26 \mathrm{a}$ & $95.76 \pm 0.96 \mathrm{~b}$ \\
\hline NZORP7 & $97.68 \pm 1.43 \mathrm{a}$ & $97.04 \pm 1.28 \mathrm{a}$ & $97.04 \pm 1.40 \mathrm{a}$ & $97.92 \pm 1.34 \mathrm{ab}$ & $97.04 \pm 1.51 \mathrm{a}$ & $97.60 \pm 1.36 \mathrm{ab}$ & $98.08 \pm 1.43 \mathrm{a}$ & $97.60 \pm 1.33 \mathrm{ab}$ \\
\hline
\end{tabular}

Table 3. Pollen germination and viability in Flower $B$ as a function of tree tomato plant material, number of days, and temperature.

\begin{tabular}{|c|c|c|c|c|c|c|c|c|}
\hline \multirow{2}{*}{$\begin{array}{l}\text { Number of days } \\
\text { Temperature }\end{array}$} & \multicolumn{2}{|c|}{ Od } & \multicolumn{2}{|c|}{$5 d$} & \multicolumn{2}{|c|}{$10 \mathrm{~d}$} & \multicolumn{2}{|c|}{$20 \mathrm{~d}$} \\
\hline & $22^{\circ} \mathrm{C}$ & $4^{\circ} \mathrm{C}$ & $22^{\circ} \mathrm{C}$ & $4^{\circ} \mathrm{C}$ & $22^{\circ} \mathrm{C}$ & $4^{\circ} \mathrm{C}$ & $22^{\circ} \mathrm{C}$ & $4^{\circ} \mathrm{C}$ \\
\hline \multicolumn{9}{|l|}{ Germination (\%) } \\
\hline CMP4 & $0.00 \pm 0.00 \mathrm{~b}$ & $5.79 \pm 7.93$ & $0.00 \pm 0.00 \mathrm{~b}$ & $0.00 \pm 0.00 \mathrm{~b}$ & $0.00 \pm 0.00 \mathrm{~b}$ & $0.00 \pm 0.00$ & $0.00 \pm 0.00$ & $0.00 \pm 0.00$ \\
\hline GT20P2 & $0.16 \pm 0.22 b$ & $0.16 \pm 0.22$ & $0.08 \pm 0.18 b$ & $0.16 \pm 0.22 b$ & $0.00 \pm 0.00 \mathrm{~b}$ & $0.40 \pm 0.49$ & $0.08 \pm 0.18$ & $0.00 \pm 0.00$ \\
\hline GT20P7 & $12.06 \pm 2.02 \mathrm{a}$ & $7.29 \pm 1.19$ & $6.45 \pm 1.71 \mathrm{a}$ & $8.99 \pm 1.09$ a & $0.24 \pm 0.22 \mathrm{a}$ & $0.16 \pm 0.22$ & $0.00 \pm 0.00$ & $0.00 \pm 0.00$ \\
\hline GT33P5 & $0.40 \pm 0.28 \mathrm{~b}$ & $0.16 \pm 0.22$ & $0.00 \pm 0.00 \mathrm{~b}$ & $0.24 \pm 0.22 b$ & $0.00 \pm 0.00 \mathrm{~b}$ & $0.40 \pm 0.89$ & $0.00 \pm 0.00$ & $0.00 \pm 0.00$ \\
\hline GT7P47 & $0.00 \pm 0.00 \mathrm{~b}$ & $5.92 \pm 8.14$ & $0.00 \pm 0.00 \mathrm{~b}$ & $0.00 \pm 0.00 \mathrm{~b}$ & $0.00 \pm 0.00 \mathrm{~b}$ & $0.00 \pm 0.00$ & $0.00 \pm 0.00$ & $0.00 \pm 0.00$ \\
\hline GT7P48 & $0.00 \pm 0.00 \mathrm{~b}$ & $5.82 \pm 5.49$ & $0.00 \pm 0.00 \mathrm{~b}$ & $0.00 \pm 0.00 \mathrm{~b}$ & $0.00 \pm 0.00 \mathrm{~b}$ & $0.00 \pm 0.00$ & $0.00 \pm 0.00$ & $0.00 \pm 0.00$ \\
\hline GT9P18 & $0.46 \pm 0.46 \mathrm{~b}$ & $0.24 \pm 0.22$ & $0.00 \pm 0.00 \mathrm{~b}$ & $0.23 \pm 0.21 \mathrm{~b}$ & $0.00 \pm 0.00 \mathrm{~b}$ & $0.00 \pm 0.00$ & $0.00 \pm 0.00$ & $0.00 \pm 0.00$ \\
\hline NZLRP5 & $1.96 \pm 2.69 \mathrm{~b}$ & $2.52 \pm 5.63$ & $0.00 \pm 0.00 \mathrm{~b}$ & $0.00 \pm 0.00 \mathrm{~b}$ & $0.00 \pm 0.00 \mathrm{~b}$ & $0.00 \pm 0.00$ & $0.00 \pm 0.00$ & $0.00 \pm 0.00$ \\
\hline NZORP7 & $2.79 \pm 3.84 \mathrm{~b}$ & $5.07 \pm 6.95$ & $0.00 \pm 0.00 \mathrm{~b}$ & $0.00 \pm 0.00 \mathrm{~b}$ & $0.00 \pm 0.00 \mathrm{~b}$ & $0.00 \pm 0.00$ & $0.00 \pm 0.00$ & $0.00 \pm 0.00$ \\
\hline \multicolumn{9}{|l|}{ Viability (\%) } \\
\hline CMP4 & $0.00 \pm 0.00 \mathrm{~b}$ & $0.00 \pm 0.00 \mathrm{~b}$ & $0.00 \pm 0.00 \mathrm{~b}$ & $0.00 \pm 0.00 \mathrm{~b}$ & $0.00 \pm 0.00 \mathrm{~b}$ & $0.00 \pm 0.00 \mathrm{~b}$ & $0.00 \pm 0.00 \mathrm{~b}$ & $0.00 \pm 0.00$ \\
\hline GT20P2 & $0.24 \pm 0.22 b$ & $0.08 \pm 0.17 b$ & $0.16 \pm 0.22 b$ & $0.32 \pm 0.33 \mathrm{~b}$ & $0.08 \pm 0.18 \mathrm{~b}$ & $0.16 \pm 0.22 \mathrm{ab}$ & $0.16 \pm 0.22 \mathrm{a}$ & $0.00 \pm 0.00$ \\
\hline GT20P7 & $11.96 \pm 2.25 \mathrm{a}$ & $7.00 \pm 1.12 \mathrm{a}$ & $5.84 \pm 2.13 \mathrm{a}$ & $7.73 \pm 1.51 \mathrm{a}$ & $0.24 \pm 0.22 b$ & $0.24 \pm 0.22 \mathrm{a}$ & $0.00 \pm 0.00 \mathrm{~b}$ & $0.00 \pm 0.00$ \\
\hline GT33P5 & $0.48 \pm 0.44 \mathrm{~b}$ & $0.23 \pm 0.21 \mathrm{c}$ & $0.00 \pm 0.00 \mathrm{~b}$ & $0.16 \pm 0.22 \mathrm{~b}$ & $0.00 \pm 0.00 \mathrm{~b}$ & $0.00 \pm 0.00 \mathrm{~b}$ & $0.00 \pm 0.00 \mathrm{~b}$ & $0.00 \pm 0.00$ \\
\hline GT7P47 & $0.00 \pm 0.00 \mathrm{~b}$ & $0.00 \pm 0.00 \mathrm{~b}$ & $0.00 \pm 0.00 \mathrm{~b}$ & $0.00 \pm 0.00 \mathrm{~b}$ & $0.00 \pm 0.00 \mathrm{~b}$ & $0.00 \pm 0.00 \mathrm{~b}$ & $0.00 \pm 0.00 \mathrm{~b}$ & $0.00 \pm 0.00$ \\
\hline GT7P48 & $0.00 \pm 0.00 \mathrm{~b}$ & $0.00 \pm 0.00 \mathrm{~b}$ & $0.00 \pm 0.00 \mathrm{~b}$ & $0.00 \pm 0.00 \mathrm{~b}$ & $0.00 \pm 0.00 \mathrm{~b}$ & $0.00 \pm 0.00 \mathrm{~b}$ & $0.00 \pm 0.00 \mathrm{~b}$ & $0.00 \pm 0.00$ \\
\hline GT9P18 & $0.24 \pm 0.22 b$ & $0.32 \pm 0.18 b$ & $0.00 \pm 0.00 \mathrm{~b}$ & $0.32 \pm 0.53 \mathrm{~b}$ & $0.00 \pm 0.00 \mathrm{~b}$ & $0.00 \pm 0.00 \mathrm{~b}$ & $0.00 \pm 0.00 \mathrm{~b}$ & $0.00 \pm 0.00$ \\
\hline NZLRP5 & $0.00 \pm 0.00 \mathrm{~b}$ & $0.00 \pm 0.00 \mathrm{~b}$ & $0.00 \pm 0.00 \mathrm{~b}$ & $0.00 \pm 0.00 \mathrm{~b}$ & $0.00 \pm 0.00 \mathrm{~b}$ & $0.00 \pm 0.00 \mathrm{~b}$ & $0.00 \pm 0.00 \mathrm{~b}$ & $0.00 \pm 0.00$ \\
\hline NZORP7 & $0.00 \pm 0.00 \mathrm{~b}$ & $0.00 \pm 0.00 \mathrm{~b}$ & $0.00 \pm 0.00 \mathrm{~b}$ & $0.00 \pm 0.00 \mathrm{~b}$ & $0.00 \pm 0.00 \mathrm{~b}$ & $0.00 \pm 0.00 \mathrm{~b}$ & $0.00 \pm 0.00 \mathrm{~b}$ & $0.00 \pm 0.00$ \\
\hline
\end{tabular}

Table 4. Germination and viability in Flower $\mathrm{C}$ of pollen as a function of plant material of tree tomato, number of days and temperature.

\section{Conclusions}

According to the results obtained in the pollen quality tests, it was observed that in flowers $A$ and $B$, the commercial materials (CMP4, NZLRP5, and NZORP7) showed higher germination percentages than the segregants at a temperature of $4{ }^{\circ} \mathrm{C}$ in the different storage times. Only the segregants GT7P47 and GT7P48 showed germination values similar to those obtained by commercial materials at $22^{\circ} \mathrm{C}$ and $4^{\circ} \mathrm{C}$ after 0 days of storage. Regarding viability, high percentages (greater than 95\%) were observed in flowers A and B, at both temperatures and at all storage times. Consequently, the materials mentioned above would be the best options for parents in future crossing plans in the tree tomato breeding program.

On the other hand, it is concluded that tree tomato pollen can be collected in flower A or flower B and stored at $4^{\circ} \mathrm{C}$ for up to 5 days. However, it is recommended to use the pollen to make crosses immediately after it's obtained from the flower because, in this stage, it has a higher percentage of germination and high viability.

\section{Acknowledgments}

The authors thank the National Institute for Agricultural Research (INIAP) and the Spanish Agency for Development Cooperation (AECID) for their support and funding to carry out this research and Gabriela Altaminate for her participation in data collection.

\section{Bibliographic references}

1. Pringle GJ, Murray BG. Interspecific hybridisation involving the tamarillo, Cyphomandra betacea (Cav.) Sendt. (Solanaceae). N Z J Crop Horticul Sci. 1991;119(2):103-111.

2. Mertz C, Gancel A, Gunata Z, Alter P, Dhuique-Mayer C, Vaillant F, Perez A, Ruales J, Brat P. Phenolic compounds, carotenoids and antioxidant activity of three tropical fruits. J Food Compos Anal. 2009;22: 381-387.

3. Espín S, Gonzalez-Manzano S, Taco V, Poveda C, Ayuda-Durán B, Gonzalez-Paramas AM, Santos-Buelga C. Phenolic composition and antioxidant capacity of yellow and purple-red Ecuadorian cultivars of tree tomato (Solanum betaceum Cav.). Food Chem. 2016;194:1073-1080. 
4. SINAGAP. Cuadro de superficie, producción y rendimiento de tomate de árbol [Internet]. 2016. [Consultado 3 Jan 2021]. Retrieved from: http://sipa.agricultura.gob.ec/index.php/compendio-agro

5. Viera W, Sotomayor A, Tamba MV, Vásquez-Castillo W, Martínez A, Viteri P, Ron L. Estimación de parámetros de calidad del fruto para segregantes interespecíficos de tomate de árbol (Solanum betaceum Cav.) en respuesta de resistencia a la antracnosis (Colletotrichum acutatum J.H. Simmonds). Acta Agron. 2016;65(3): 301-311.

6. Torres, A. Caracterización física, química y compuestos bioactivos de pulpa madura de tomate de árbol (Cyphomandra betacea) (Cav.) Sendtn. Arch Latinoam Nutr. 2012;6(4):381-388.

7. Feicán C, Encalada C, Becerril A. Descripción agronómica del cultivo del tomate de árbol (Solanum betaceum Cav.). Agroproductividad. 2016;9(8): 78-86.

8. Villares MX, Sánchez JA, Viera WF, Soria NA, Sotomayor AV, Yánez DF, Martínez, EO. Caracterización morfológica de frutos de tomate de árbol (Solanum betaceum cav.) de una población. Rev Investig Talent. 2018;5(1): 9-19.

9. Viera W, León J, Ochoa J. Evaluación de fungicidas in vitro y pruebas de resistencia de cinco variedades de tomate de árbol (Solanum betaceum Cav.) para antracnosis (Colletotrichum gloeosporioides) Cutulagua, Pichincha. Rev Rumipamba. 2002;16(1):89-91.

10. Viera W, Sotomayor A, Viteri P. 2019. Breeding of three Andean fruit crops in Ecuador. Chron Hortic. 2019;59(4):20-29.

11. Benítez E, Viera W, Garrido P, Flores F. Current research on Andean fruit crop diseases. Chong-Aguirre PA, Newman DJ, Steinmacher DA, editors. Agricultural, Forestry and Bioindustry Biotechnology and Biodiscovery. Switzerland: Springer; 2020. p. 387-401.

12. Carrillo E, Aller A, Cruz S, Álvarez J. Andean berries from Ecuador: A re-view on botany, agronomy, chemistry and health potential. J Berry Res. 2015;5(2)49-69.

13. Ordoñez RM, Cardozo ML, Zampini IC, Isla MI. Evaluation of antioxidant activity and genotoxicity of alcoholic and aqueous beverages and pomace derived from ripe fruits of Cyphomandra betaceum Sendt. J Agric Food Chem. 2010;58:331-3337.

14. Moreno Alvarez M, Girán N, Serrano K, García D, Camacho, D. Evaluación microbiologica y fisicoquímica de néctares pasteurizados elaborados con pulpa de tomate de árbol (Cyphomandra betaceae Sendth). Arch Latinoam Nutr. 2003;53(3), 282-286.

15. Ramírez-Godina F, Robledo-Torres V, Foroughbakhch-Pournavab R, Benavides-Mendoza A, Alvarado-Vázquez M. Viabilidad de polen, densidad y tamaño de estomas en autotetraploides y diploides de Physalis ixocarpa. Botanical Sciences 2013;91(1):11-18.

16. Gonzáles M, Estévez A, Castillo J, Salomón J, More O, Hernández M. La calidad del polen: Prerrequisito indispensable del mejoramiento tradicional de la papa en Cuba. Rev Latinoam Papa. 2002;13(1):75-94.

17. Araméndiz H, Cardona C, Lugo A. germinación del polen de berenjena (Solanum melongena L.) en condiciones in vitro. Rev Fac Nal Agr Medellín. 2012;65(2), 6637-6643.

18. Abreu I, Costa M, Oliviera M, Cunha M, Castro R. Ultrastructure and germination of Vitis vinifera cv. Loureiro pollen. Protoplasma. 2006;228:131-135.

19. Salles L, Ramos J, Pasqual M, Junqueira K, Bortolotti da Silva A. Sacarose e pH na germinação in vitro de grãos de pólen de citros. Cienc Agrotecnologia. 2006;30(1):170-174.

20. Ordoñez, B. Determinación de la viabilidad y fertilidad del polen. Centro Lima, Perú: Internacional de la Papa; 2014.
21. Marks GE. 1954. An aceto-carmine glycerol jelly for use in pollen fertility counts. Stain Technol. 1954;29(5):277.

22. Rodriguez T, Dafni A. 2000. A new procedure to asses pollen viability. Sex Plant Reprod. 2000;12(4):241-244.

23. Singh I, Shono M. Effect of 24-epibrassinolide on pollen viability during heat-stress in tomato. Indian $\mathrm{J}$ Experim Biol. 2003:41(2):174-176.

24.França-Neto LV, Nascimento W, Carmona RE, De Freitas R. Viability of eggplant pollen. Crop Breeding Appl Biotechn. 2009;9(4):320-327.

25. Suárez L, Castilla Y, Hernández M, Salomón J, Estévez A, Céspedes $\mathrm{O}$, Araujo B. Efecto del PECTIMORF ${ }^{\circledR}$ en la germinación in vitro del polen de papa (Solanum tuberosum). Temas Cienc Tecn. 2010;14(40):43-46.

26.Grisales-Vásquez N, Trillos-Gonzáles O, Cotes-Torres J, Orozco-Orozco L. Estudios de fertilidad de polen en accesiones de uchuva (Physalis peruviana L.). Rev Fac Cienc Basic Univ Mil Nueva Granada. 2010;6(1):42-51.

27. Stone JL, Thomson JD, Dent-Acosta SJ. Assessment of pollen viability in hand-pollination experiments: A review. Am Jour Botany. 1995;82(9): 1186-1197.

28. Revelo J, Pérez E, Maila M. Cultivo ecológico de tomate de árbol en Ecuador. Quito, Ecuador: INIAP; 2004.

29.González ME, Estévez A, Rodríguez T., Álvarez M. Estudio de la fertilidad del polen en especies de papa. Cultivos Trop. 1992;13:70-73

30.Lagos T, Caetano C, Alirio-Vallejo F, Muñoz J, Criollo H, Olaya C. Caracterización palinológica y viabilidad polínica de Physalis peruviana L. y Physalis philadelphica Lam. Agron Colomb. 2005;23(1),55-61.

31. Montaner C, Floris E, Álvarez JM. Study of pollen cytology and evaluation of pollen viability using in vivo and in vitro test, in borage (Borago officinalis L.). Grana. 2003;42: 33-37.

32. Atiencia, V. Estudio de la viabilidad del polen de chirimoya (Annona cherimola Mill) almacenado en condiciones ambientales y controladas, como base para la polinización manual en la Granja Tumbaco del Programa de Fruticultura del INIAP, Tumbaco - ECuador. Tesis previa a la obtención del título de Ingeniero Agrónomo. Universidad Estatal de Bolívar. Facultad de Ciencias Agropecuarias, Recursos Naturales y del Ambiente; 2010.

Received: 26 February 2021

Accepted: 20 August 2021 